\title{
Intracranial haemorrhage and the phenomenon of 'T2-blackout'
}

\author{
${ }^{1} D J$ van der Merwe, $M B C h B$ \\ ${ }^{1} S$ Andronikou, MB BCh, FCRad, FRCR, PhD \\ ${ }^{2} S$ McGurck, FRCR \\ ${ }^{1} S$ Hlongwane, $M B C h B$ \\ ${ }^{1}$ A Brandt, $M B C h B$ \\ ${ }^{1}$ Department of Radiology, Stellenbosch University, Tygerberg \\ ${ }^{2}$ Royal Hospital for Sick Children, Edinburgh, UK
}

Diffusion-weighted imaging (DWI) and corresponding apparent diffusion coefficient (ADC) maps have become routine magnetic resonance imaging (MRI) sequences. In children one of the major uses of DWI is in neonates with perinatal hypoxic ischaemic injury. ${ }^{1}$ Premature patients with hypoxic events, however, are also susceptible to intracranial haemorrhage. It is useful to be aware of the signal changes of the different stages of haemorrhage on DWI.

Our patient was born post term via caesarian section because of fetal heart rate abnormalities. He had a low birth weight and meconium at delivery. Initially he was hypertonic with hypotonia in the subsequent stages after birth. MRI was performed at 9 days.

There was a haematoma adjacent to the left trigone which was hyperintense on T1 (Fig. 1), hypointense on T2 (Fig. 2). It showed 'blackout'

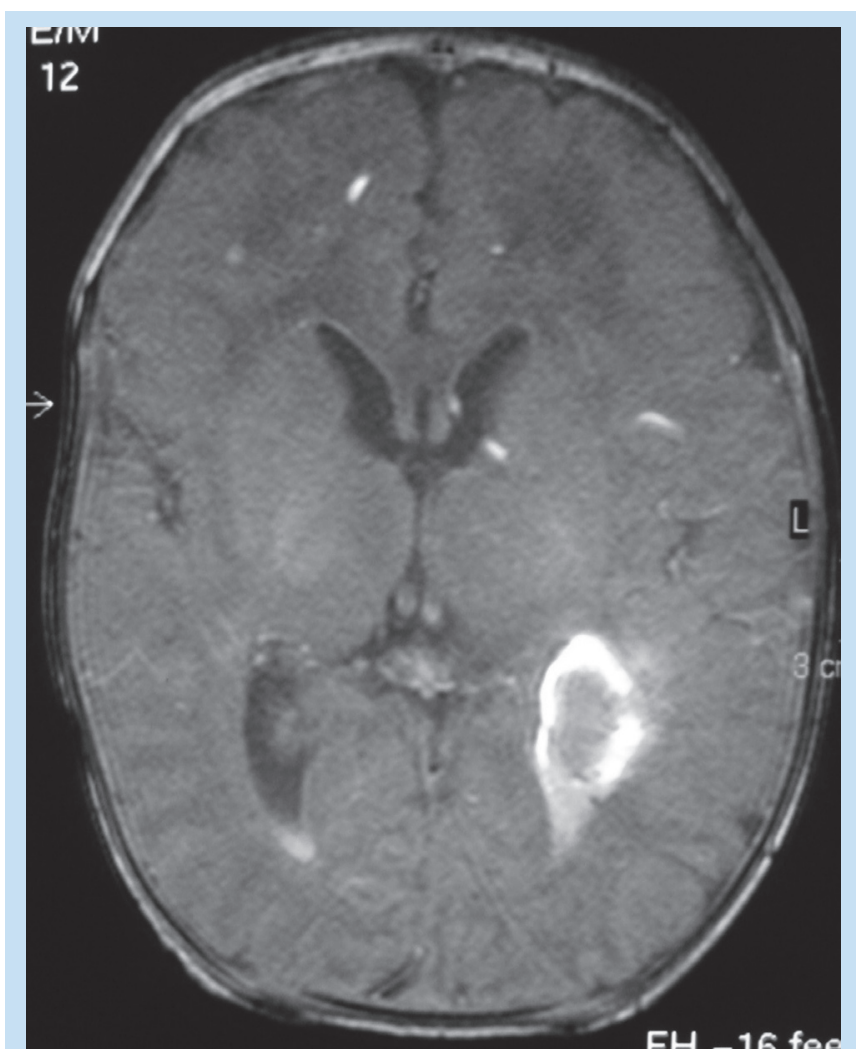

Fig 1. There is a haematoma adjacent to the left trigone that is hyperintense on T1. Blood is also present within the lateral ventricle.

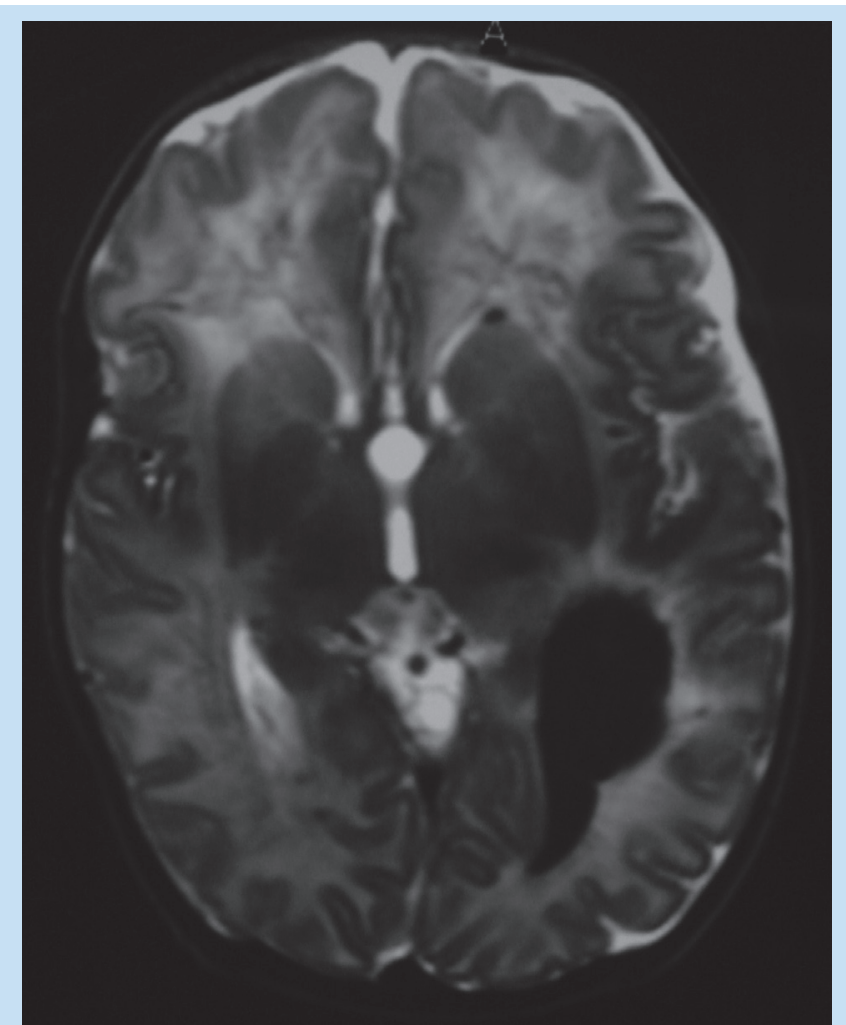

Fig. 2. The haematoma is hypointense on T2. Also note the hyperintense periventricular white matter representing hypoxic injury.

(hypointense) on DWI (Fig. 3) and ADC map (Fig. 4). Periventricular white matter showed high signal on T2, which showed restricted diffusion with high signal on DWI (Fig. 3) and low signal on ADC map (Fig. 4). This is consistent with a global hypoxic injury.

Hyperacute haematomas are hyperintense on T2-weighted imaging and DWI because of the T2 shine-through phenomenon. ${ }^{2} \mathrm{~A}$ less well-known phenomenon is termed 'T2- blackout' which occurs in the acute and early subacute stage when haematomas are hypointense on T2-weighted imaging. These haematomas are equally hypointense on DWI and ADC map: T2-blackout. ${ }^{3}$

1. Oksuzler YF, Cakmakci H, Kurul S, Oksuzler M, Dirik E. Diagnostic value of diffusion-weighted magnetic resonance imaging in pediatric cerebral diseases. Pediatr Neurol 2005; 32: 325-333.

2. Moritani T, Ekholm S, Westesson PL. Pitfalls and artifacts of DWI imaging. In: Diffusion Weighted MR Imaging of the Brain. Heidelberg: Springer, 2005: 12-17.

3. Silvera S, Oppenheim C, Touze E, et al. Spontaneous intracerebral hematoma on diffusion-weighted images: Influence of T2-shine-through and T2-blackout effects. AJNR 2005; 26: 236-241. 


\section{PICTORIAL INTERLUDE}

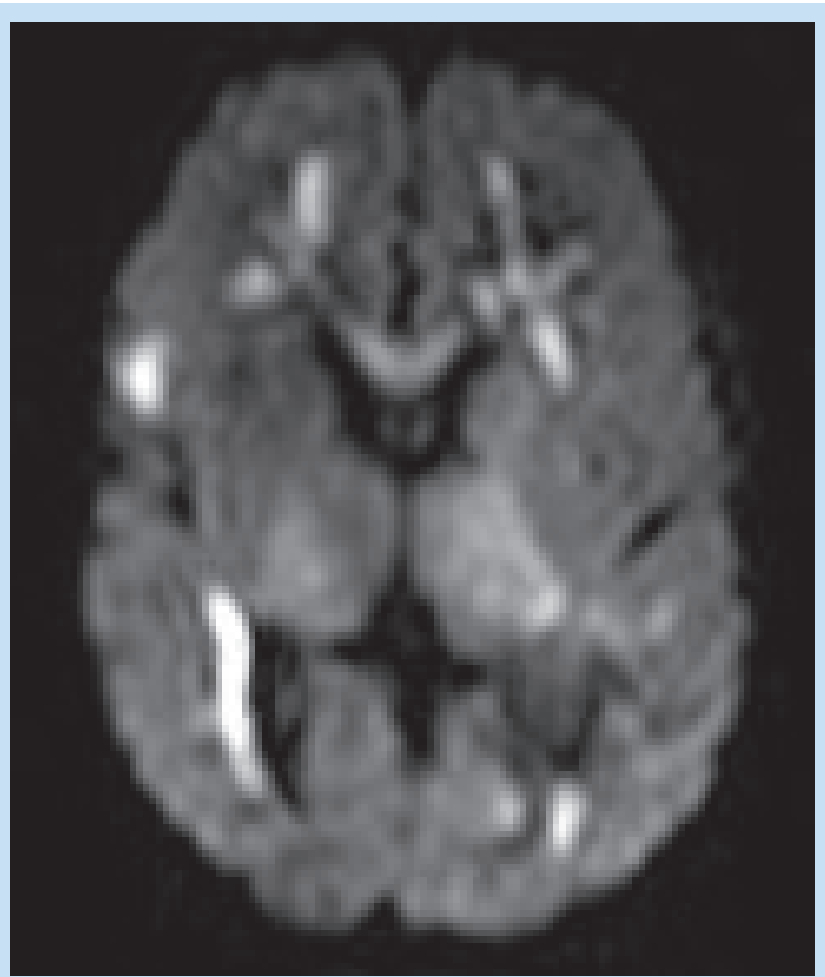

Fig. 3. DWI (B 1000). There is high signal in the periventricular white matter and thalami consistent with hypoxic injury. The haematoma and intraventricular blood on the left is hypointense: 'blackout'

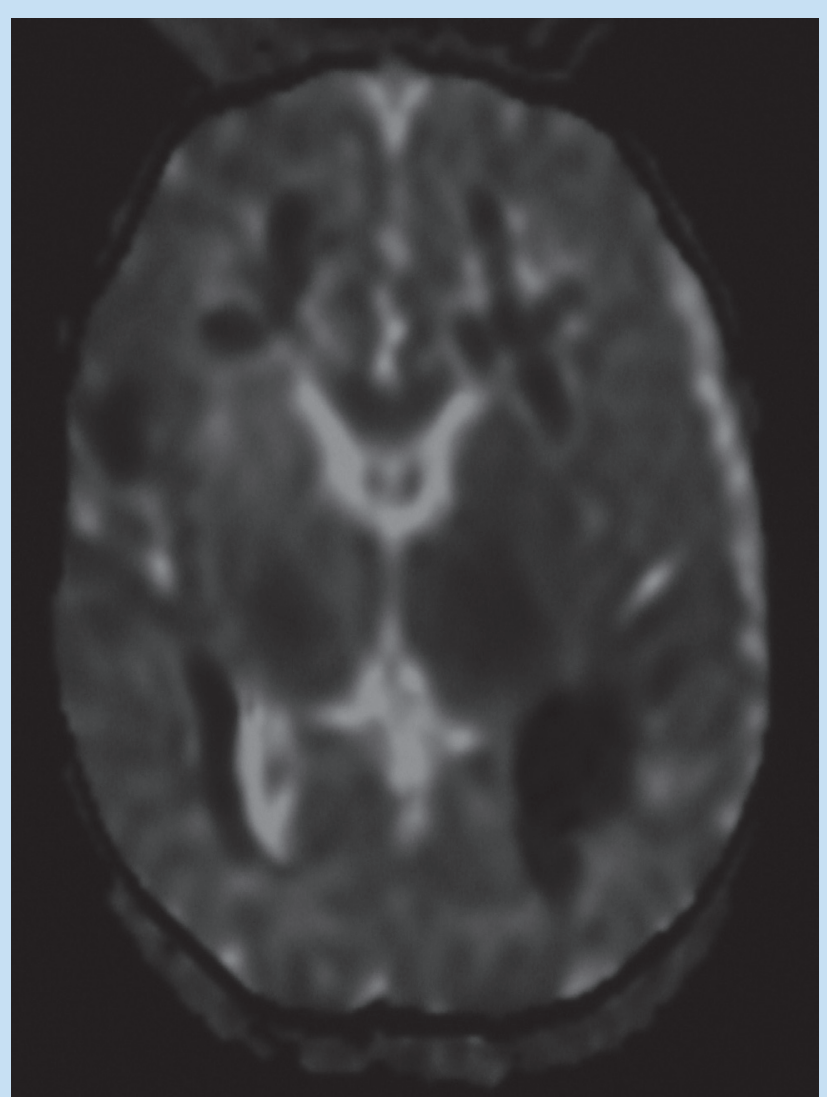

Fig. 4. There is a hypointense area on ADC map in the area of the haematoma ('blackout'). Also note the low density in the periventricular white matter consistent with restricted diffusion.

\section{Erratum}

In the case report 'Radiological features of simple (unicameral) bone cysts' in the September 2007 issue of the SA Journal of Radiology (p. 63) Figs. 2 and 3 were placed incorrectly. Please see corrected figures below.

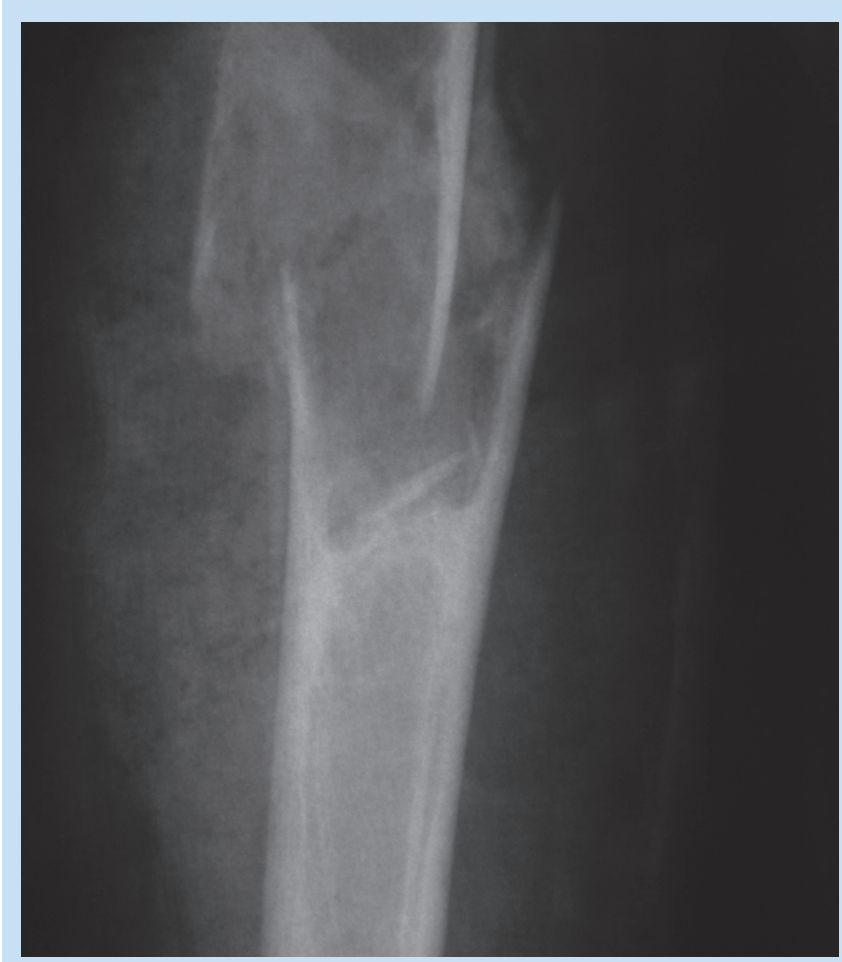

Fig. 2. In addition to the visualised pathological fracture, a 'fallen fragment' is demonstrated which is almost pathognomonic of a simple bone cyst.

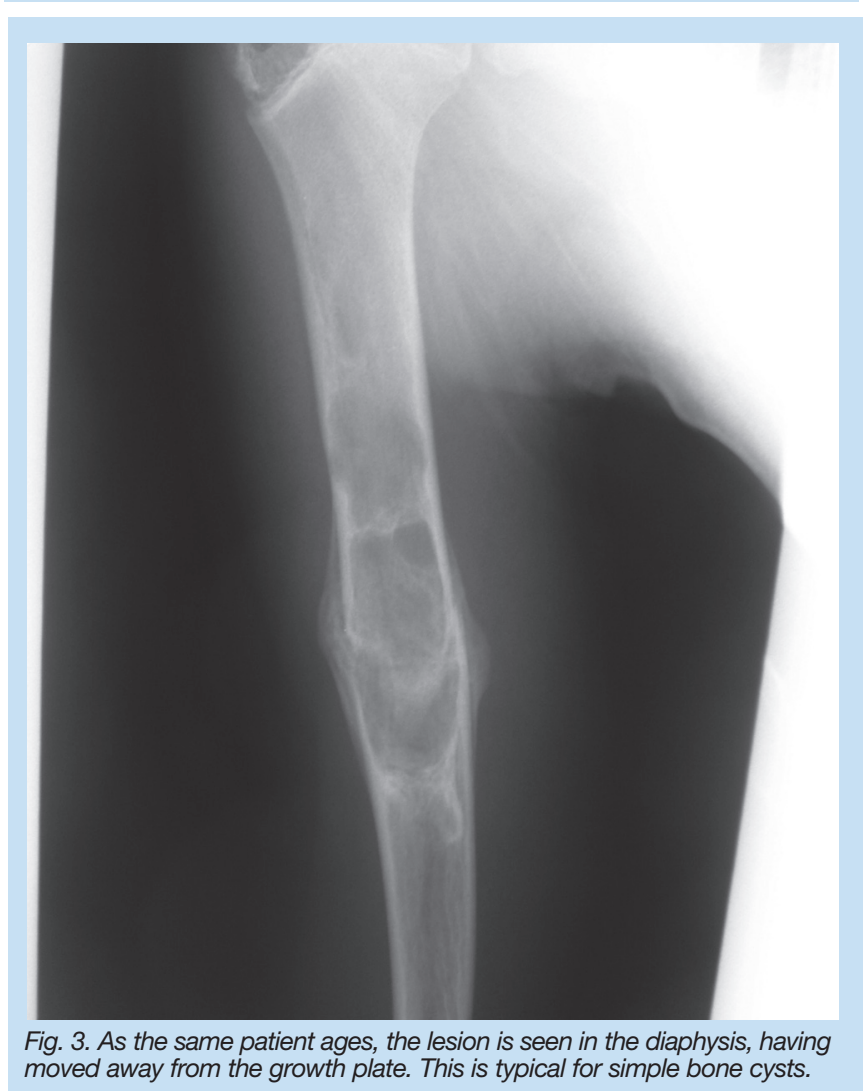

